\title{
Deletion of the receptor tyrosine kinase Tyro3 inhibits synovial hyperplasia and bone damage in arthritis
}

\author{
Gisela Ruiz-Heiland, ${ }_{1}^{1}$ Yi Zhao, ${ }^{2}$ Anja Derer, ${ }^{1}$ Tobias Braun, ${ }^{1}$ Klaus Engelke, ${ }^{3}$ \\ Elena Neumann, ${ }^{4}$ Ulf Mueller-Ladner, ${ }^{4}$ Yi Liu, $^{2}$ Jochen Zwerina, ${ }^{1,5}$ Georg Schett ${ }^{1}$
}

\begin{abstract}
Handling editor Tore K Kvien
- Additional material is

published online only. To view please visit the journal online (http://dx.doi.org/10.1136/ annrheumdis-2012-202907).

${ }^{1}$ Department of Internal Medicine 3, Institute for Clinical Immunology, University of Erlangen-Nuremberg, Erlangen, Germany

${ }^{2}$ Department of Rheumatology and Immunology, West China Hospital, Sichuan University, Chengdu, China

${ }^{3}$ Institute of Medical Physics University of ErlangenNurnberg, Erlangen, Germany ${ }^{4}$ Department of Rheumatology, University of Giessen, Bad Nauheim, Germany ${ }^{5}$ Ludwig Boltzmann Institute of Osteology, Hanusch Hospital, Vienna, Austria
\end{abstract}

\section{Correspondence to} Professor Georg Schett, Department of Internal Medicine 3 and Institute for Clinical Immunology, University of Erlangen-Nuremberg, Krankenhausstrasse 12, Erlangen D-91054, Germany; georg.schett@uk-erlangen.de

Accepted 1 April 2013 Published Online First 30 April 2013

\footnotetext{
To cite: Ruiz-Heiland $\mathrm{G}$,

Zhao Y, Derer $A$, et al. Ann Rheum Dis 2014;73:

771-779.
}

\section{ABSTRACT}

Objective To test whether the tyrosine kinase Tyro3 affects arthritis. Tyro3, the ligand of growth arrest-specific protein 6 (GAS6) is a receptor tyrosine kinase involved in cell survival. Tyro3 and GAS6 are expressed in the arthritic synovium, and in vitro studies have shown their role in osteoclast differentiation.

Methods Bone was assessed by micro CT and histomorphometry in Tyro3-deficient $\left(\mathrm{TyrO}^{-1-}\right)$ and wild-type mice. Arthritis was induced in both genotypes, and Gas6 level was measured by ELISA. Synovitis, synovial hyperplasia, bone erosion, osteoclast activation and osteoclast gene expression were assessed by histomorphometry and reverse transcriptase-PCR, respectively. In vitro osteoclast differentiation assays were performed in $\mathrm{Tyro3}^{-/-}$and wild-type mice. Furthermore, effects of Tyro3 and GAS6 on human synovial fibroblast proliferation and osteoclastogenesis were assessed in human cells.

Results $\mathrm{TyrO3}^{-1-}$ mice had significantly higher bone mass than wild-type littermates. Induction of arthritis increased GAS6 serum levels. Arthritic Tyro3 ${ }^{-1-}$ mice showed less synovial hyperplasia, osteoclast numbers and bone damage compared with controls. In vivo expression of osteoclast-associated receptor and receptor activator of nuclear factor $-\kappa B$ and in vitro osteoclastogenesis were impaired in $\mathrm{Trro}^{-1-}$ mice. GAS6 also induced synovial fibroblast proliferation and osteoclast differentiation in human cells in

Tyro3-dependent manner.

Conclusions These findings indicate that Tyro3 is a critical signal for synovial hyperplasia, osteoclast differentiation and bone erosion during arthritis. GAS6 and Tyro3 therefore constitute therapeutic targets to inhibit synovial hyperplasia and associated bone erosion.

\section{INTRODUCTION}

Receptor tyrosine kinases (RTKs) are plasma membrane receptors, which essentially mediate the effect of growth factor on cells. Examples for RTKs include the insulin receptor as well as the receptors for vascular endothelial growth factor and plateletderived growth factor. A less known RTK is Tyro3, which is expressed on the plasma membrane of a variety of different cells and acts as receptor for growth arrest-specific protein 6 (GAS6). ${ }^{1}$ Tyro 3 is not the only receptor for GAS6, which also activates the receptor tyrosin kinases Axl and Mer, transmitting anti-apoptotic and survival signals to cells. $^{2}$ Activation of Tyro3 by GAS6 binding controls a variety of cellular processes such as tolllike receptor (TLR)-mediated and type I interferonmediated regulation of cytokine production, ${ }^{3-5}$ haematopoiesis, ${ }^{6}$ thrombus formation ${ }^{7}$ and cell proliferation. ${ }^{8}$ Both Tyro3 and GAS6 are expressed in the synovial membrane of patients with rheumatoid arthritis (RA) and appear to play a role in the survival of endothelial cells in the synovial tissue of RA patients. ${ }^{9}$ Furthermore, GAS6 expression appears to be stimulated by inflammatory reaction, since increased serum levels of GAS6 have been described in sepsis and other types of systemic inflammatory responses. ${ }^{10}$ Interestingly, there is also a role of Tyro3 in bone metabolism: binding of GAS6 to Tyro3 leads to enhanced differentiation of bone-forming osteoclasts. ${ }^{11-13}$

RA is characterised by chronic inflammation and hyperplasia of the synovial membrane, which leads to progressive destruction of bone and the adjacent articular cartilage. Inflammatory cytokines are considered as the essential triggers for bone loss in RA. ${ }^{14}$ Bone destruction in RA is closely linked to the presence of an activated synovial inflammatory tissue as well as synovial hyperplasia, which provides the essential signals for the development of bone-resorbing osteoclasts. ${ }^{16} 17$ Synovial-derived molecules, which trigger the generation of boneresorbing osteoclasts, include inflammatory cytokines such as tumour necrosis factor-alpha (TNF- $\alpha$ ), interleukin (IL)-6/IL-6R complexes and IL-1 as well as essential growth and differentiation factors for osteoclasts such as macrophage colony-stimulating factor (M-CSF) and receptor activator of nuclear factor- $\kappa \mathrm{B}$ ligand (RANKL). ${ }^{18}$

GAS6-Tyro3 interactions may be an interesting link between synovial inflammation and bone loss for several reasons: (1) GAS6 as well as its receptor Tyro3 are expressed in the synovial membrane of patients with $\mathrm{RA}^{9}$; (2) engagement of Tyro3 by GAS6 has been identified as direct inducer for osteoclastogenesis $^{11-13}$; and (3) GAS6-Tyro3 interactions play a role in the survival of cells involved in synovial inflammation such as fibroblasts, monocytes/macrophages, endothelial cells and chondrocytes. ${ }^{4} 91920$ Apart from expression of Tyro3 and GAS6 in human RA and in vitro studies showing a role of GAS6-Tyro3 interactions in osteoclast differentiation, nothing is know about a potential in vivo role of the GAS6-Tyro3 system in arthritis. Based on the function of Tyro3, we hypothesised that Tyro3 activation may play a key role in 
inflammatory bone loss. To test this hypothesis, we assessed the bone phenotype of mice deficient for Tyro3 $\left(\mathrm{Tyro}^{-1-}\right.$ ) and also induced arthritis in these mice and respective controls to analyse inflammatory bone changes.

\section{MATERIAL AND METHODS \\ Animals}

Mice deficient for Tyro3 (Tyro3 $^{-/-}$) (B6. 129-Tyro3 ${ }^{\text {tm1GRL }} / \mathrm{J}$ ) has been described previously ${ }^{5}$ and were purchased from Jackson Laboratory (Bar Harbour, Maine, USA). Mice were backcrossed into the $\mathrm{C} 57 \mathrm{Bl} 6$ background for six generations. Littermate wild-type mice expressing Tyro3 $\left(\right.$ Tyro $3^{+/+}$) were used as controls for all the experiments. Approval for all animal procedures was obtained by the ethical committee of the University of Erlangen-Nuremberg.

\section{Arthritis model}

Eight-week-old Tyro3-deficient animals $(n=10)$ and littermate wild-type controls $(n=10)$ were used for induction of arthritis. Two independent experiments with each five wild-type and five mutant mice were conducted. Arthritis was induced by intraperitoneal injection of $250 \mu \mathrm{l}$ serum obtained from $\mathrm{K} / \mathrm{BxN}$ mice as described previously. ${ }^{21}$ Disease progression was assessed by measuring joint swelling, grip strength and body weight every other day. After 12 days, animals were scarified by cervical dislocation.

\section{Paw histology}

Hind paws were fixed overnight in 4\% paraformaldehyde and decalcified using EDTA. The material was then embedded in paraffin, cut and $1-2 \mu \mathrm{m}$ sections were stained with $\mathrm{H} \& \mathrm{E}$, toluidine blue or tartrate-resistant acid phosphatase (TRAP) for histological evaluation of articular inflammation, proteoglycan loss, bone erosions and osteoclast assessment. Analysis was performed using a microscope (Nikon, Berlin, Germany) equipped with a digital camera and digital analysis system (OsteoMeasure; OsteoMetrics, Decatur, Georgia, USA).

\section{Histomorphometry}

Ten-week-old Tyro3 $^{-/-}$mice $(\mathrm{N}=10)$ and 10 wild-type littermate controls were sacrificed, and tibial bones were prepared and fixed overnight with $4 \%$ paraformaldehyde. Right tibial bones were embedded in methylmetacrylate (Technovit; Heraeus Kulzer, Wehrheim, Germany), cut and stained by Goldner trichrome and Van Kossa for histomorphometry. Bone histomorphometry was performed using a microscope (Nikon, Japan) equipped with a video camera and digital analysis system (OsteoMeasure; OsteoMetrics, Decatur, California, USA). The fraction of bone volume of the total sample volume (bone volume per total volume (BV/TV)), trabecular number (Tb. N), trabecular thickness (Tb. Th), trabecular separation (Tb.Sp), number of osteoclasts per bone perimeter (N.Oc/B.Pm), number of osteoblasts per bone perimeter (N.Ob/B.Pm) were measured according to international standards. ${ }^{22}$ In vivo bone formation was assessed by dynamic histomorphometry after injecting $0.6 \mathrm{mg}$ calcein (Sigma-Aldrich, St Louis, Missouri, USA) subcutaneously 9 and 2 days before sacrificing the mice. The mineral apposition rate (MAR; $\mu \mathrm{m} /$ day) was determined by fluorescent microscopy. Left tibias were decalcified with EDTA and stained using TRAP staining for osteoclast assessment.

\section{Micro-computed tomography}

Tibias were analysed by CT using a high-resolution CT scanner (GE explore Locus SP Specimen Scanner; GE Healthcare,
London, Canada). Bones were placed in a sealed acrylic specimen holder containing phosphate-buffered saline and a hydroxyapatite phantom, which was used to calibrate Hounsfield units to mineral density $(\mathrm{mg} / \mathrm{ml})$. After scanning, images were reconstructed to an isotropic voxel size of 13.2 $\mu^{3}$ using a standard Feldkamp Conebeam algorithm and a threshold of $585 \mathrm{mg} / \mathrm{ml}$. The following parameters were analysed: bone volume per total volume (BV/TV), Tb.Th, Tb.N and Tb.Sp.

\section{Serum analyses}

Serum was analysed using commercially available ELISA kits for Rat Laps (IDS Immunodiagnostic Systems), TRAP 5b (IDS Immunodiagnostic Systems) and GAS6 (AB Adipobiotech).

\section{Osteoclast differentiation assay}

Bone marrow cells were isolated and cultured for 3 days in $50 \mathrm{ng} / \mathrm{ml}$ of M-CSF. Cells $\left(1 \times 10^{6} /\right.$ well $)$ were plated on a 24-wells cell culture plate (Costar-Corning, New York, USA) and cultured in Dulbecco's modified Eagle's medium (DMEM) supplemented with $10 \%$ fetal calf serum, $50 \mathrm{ng} / \mathrm{ml}$ of M-CSF and $30 \mathrm{ng} / \mathrm{ml}$ of RANKL (both from R\&D Systems) for another 3-4 days. Osteoclasts were detected by staining for TRAP staining using a commercial kit (Sigma-Aldrich) following manufacturer's instructions. Osteoclasts were identified as multinucleated purple-stained cells.

\section{Real-time PCR}

RNA was isolated from arthritic knee joints or osteoclast cultures by using Trizol (Invitrogen, Grand Island, New York, USA) following manufacturer's instructions. RNA concentration was measured before genomic DNA was digested with DNAse I (Fermentas, St Leon-Rot, Germany), and cDNA was synthesised in the presence of RNAse inhibitor. For SYBR green-based detection, a dissociation curve was carried out by one cycle following the last amplification cycle to control for the specificity of PCR amplification: $95^{\circ} \mathrm{C}$ for $15 \mathrm{~s}, 60^{\circ} \mathrm{C}$ for $30 \mathrm{~s}$ and $95^{\circ} \mathrm{C}$ for $15 \mathrm{~s}$. Relative quantification was performed by calculating the difference in cross-threshold values $\left(\Delta C_{t}\right)$ of the gene of interest and a housekeeping gene according to the equation $2^{-\Delta C \mathrm{t}}$. For primers details, see online supplementary table 1 .

\section{Human osteoclast culture}

Peripheral blood mononuclear cells were isolated from $10 \mathrm{ml}$ EDTA blood of healthy donors using a Ficoll gradient (Lyphoflot, BioRad) and incubated in 48 -well plates $\left(7.5 \times 10^{5}\right.$ cells per well) in $\alpha$-minimum essential medium (MEM) (Gibco) with $1 \%$ PenStrep (Gibco). After $3 \mathrm{~h}$, the cells were washed for monocyte enrichment and cultivated for 14 days in $\alpha$-MEM supplemented with $10 \%$ heat-inactivated fetal bovine serum (Biochrom AG), 1\% penicillin/ streptomycin (Gibco), $10 \mathrm{ng} / \mathrm{ml}$ M-CSF and submaximal concentrations of $1 \mathrm{ng} / \mathrm{ml}$ RANKL (both Peprotech). Every 3 days, medium was replaced with fresh medium. Various concentrations of recombinant GAS6 (R\&D), Tyro3:Fc (also termed DTK:Fc), Axl:Fc (both Sigma Aldrich) or OPG:Fc (R\&D Systems) were added as indicated. For coculture experiments with synovial fibroblasts, osteoclasts were isolated and cultured according to the same protocol as described above with the exception that no RANKL was added. Osteoclast differentiation was evaluated by staining cells for TRAP using a Leukocyte Acid Phosphatase Kit (Sigma-Aldrich) according to the manufacturer's instructions. 


\section{Human synovial fibroblast culture}

Synovial tissue was retrieved from three patients with RA receiving synovectomy of the knee joint. Synovial tissue was minced and digested in dispase II $(1.5 \mathrm{mg} / \mathrm{ml}$; Roche Diagnostics Corporation, Indianapolis, Indiana, USA) dissolved in DMEM (without any supplements) for $2 \mathrm{~h}$ with vortexing steps each $15 \mathrm{~min}$. The tissue suspension was then filtered through a 70 $\mu \mathrm{m}$ cell strainer. The cell suspension was collected, centrifuged, resuspended and cultured in DMEM (Invitrogen, Carlsbad, California, USA) supplemented with 10\% heat-inactivated fetal bovine serum (Biochrom AG), 1\% penicillin/streptomycin (Gibco). Cells from passages 3 to 5 were used for in vitro assays. Cells were used for coculture experiments as described above and as monocultures for assessing cell proliferation. The proliferation rate was determined at passage 4 using the CyQUANT Cell Proliferation Assay Kit (Invitrogen), according to the manufacturer's instructions. Fluorescence measurements were made with excitation at $485 \mathrm{~nm}$ and emission detection at $530 \mathrm{~nm}$.

\section{Statistical analysis}

Data are presented as the mean \pm SEM. For group comparison, we used the non-parametric Mann-Whitney U test. A p value of less than 0.05 was considered significant.

\section{RESULTS}

\section{Increased bone mass in mice deficient in tyro3}

First, we analysed the bone phenotype of tyro3-deficient mice. Using 10 -week-old tyro $3^{-/-}$mice, we performed $\mu \mathrm{CT}$ examination of the tibial bones. Analysis revealed a significant increase $(p=0.031)$ in bone mass in tyro3 $3^{-1-}$ mice in comparison to tyro $^{+/+}$littermates. Bone microstructure analysis showed that increased bone mass in tyro $3^{-1}$ is based on enhanced Tb.Th, whereas Tb.N and Tb.Sp were not different among tyro3 $3^{-1-}$ mice and littermate controls (figure 1A,B).

We also performed histomorphometric analysis of trabecular bone to further define bone microstructure of tyro $3^{-/-}$mice. Similar to the data obtained from $\mu \mathrm{CT}$ measurements, bone mass (BV/TV) as well as Tb.Th were significantly increased in tyro $^{-/-}$mice compared with wild-type littermates. Evaluation of $\mathrm{Tb} . \mathrm{N}$ and separation did not show major differences between tyro $^{-/-}$mice and the controls (figure $1 \mathrm{~B}$ ). With respect to bone cells, tyro $3^{-/-}$mice and littermate controls were not different in osteoblast parameters such as osteoblast numbers and osteoblastcovered bone surface. Also, dynamic histomorphometry showed no significant difference in the MARs between the two groups (tyro $^{-/-}, 1.07 \pm 0.16 \mu \mathrm{m} /$ day; tyro $3^{+/+}, 1.15 \pm 0.09 \mu \mathrm{m} /$ day). In contrast, osteoclasts were significantly reduced in their numbers (No.c/B.Pm) in tyro3 ${ }^{-/-}$mice compared with littermate controls (figure 1C,D).

\section{Upregulation of the tyro3 ligand GAS6 in arthritis}

We next attempted to define a potential role of tyro3 in pathological bone loss, since the ligand of tyro3 and GAS6 is expressed in the joints of patients with human RA. ${ }^{9}$ We induced arthritis by injection of serum from $\mathrm{K} / \mathrm{BxN}$ mice into tyro $3^{-/-}$ mice and wild-type littermate controls and measured the serum level of GAS6. Arthritis indeed led to a significant increase in GAS6 levels in tyro3 ${ }^{-/}$mice (mean \pm SD before arthritis: 1131 $\pm 2.00 \mathrm{ng} / \mathrm{ml}$ and after arthritis: $13.51 \pm 1.65 \mathrm{ng} / \mathrm{ml}$ ) and wildtype controls (before arthritis: $10.05 \pm 1.20 \mathrm{ng} / \mathrm{ml}$ and after arthritis: $13.58 \pm 2.81 \mathrm{ng} / \mathrm{ml}$ ) (figure 2A). Moreover, GAS6 levels were comparable in tyro $3^{-1-}$ mice and wild-type controls.

\section{Attenuated arthritis and arthritis-related bone loss in tyro3-deficient mice}

Based on the phenotype of higher bone mass in conjunction with impaired bone resorption in tyro $^{-/-}$mice as well as enhanced GAS6 levels in arthritis, we evaluated whether GAS6tyro3 interaction affects bone loss during arthritis. After induction of arthritis in tyro $3^{-/-}$mice and wild-type littermates, we analysed clinical disease activity in tyro $3^{-/-}$mice and tyro $3^{+/+}$ controls. Paw swelling was milder in tyro $3^{-/-}$mice than in the controls (figure 2B). Histological analysis of the hind paws showed mild but significant reduction of synovial inflammation in tyro $3^{-/-}$mice compared with controls (figure $3 \mathrm{~A}$ and $\mathrm{B}$ ). Most strikingly, however, synovial hyperplasia was almost absent in tyro $3^{-/-}$mice (figure $3 \mathrm{~A}$ and $\mathrm{C}$ ), and significant $(\mathrm{p}=0.045)$ reduction of bone erosions was found compared with controls (figure 3D and E). Osteoclast numbers were significantly lower $(\mathrm{p}=0.049)$ in tyro $3^{-/-}$mice compared with controls (figure $3 \mathrm{~F}$ ).

To further analyse bone protection in tyro $3^{-/-}$mice during arthritis, we measured systemic bone resorption in the serum of tyro $^{-/-}$mice as well as local expression of osteoclast-specific markers in the joints. In accordance with histological findings, serum level of collagen type I cleavage products, a marker for bone resorption, was significantly $(\mathrm{p}=0.015)$ lower in tyro3 ${ }^{-/-}$ mice $(41.5 \pm 6.8 \mathrm{ng} / \mathrm{ml})$ compared with wild-type controls $(89.55 \pm 54.27 \mathrm{ng} / \mathrm{ml})$. Similar results were obtained for the serum levels of matrix metalloproteinase 3 (figure $3 \mathrm{G}$ ). Furthermore, we analysed the expression of osteoclast genes in the arthritic joints of tyro $3^{-/-}$mice and respective controls by real-time PCR analysis. We could show significantly lower expression of two essential receptors for osteoclast activation, OSCAR and, in tyro $3^{-/}$arthritic mice compared with littermate controls (figure $3 \mathrm{H}$ ).

\section{Impaired osteoclast differentiation in tyro3 $3^{-1-}$ mice}

We next tested whether tyro $3^{-/-}$monocytes show an intrinsic defect to differentiate into osteoclasts. Monocytes were isolated from the bone marrow of tyro $3^{-/-}$-deficient mice and littermate wild-type controls and differentiated into osteoclast in vitro. Osteoclast differentiation was significantly impaired in monocytes of tyro $3^{-/-}$-deficient mice compared with those from wildtype controls. The number of multinucleated TRAP-positive cells, resembling osteoclasts, which could be generated from bone marrow monocytes of tyro $3^{-/-}$-deficient mice was significantly lower than in wild-type littermate controls (figure 4A,B). When analysing the expression of the osteoclast receptors, RANK and OSCAR, which were downregulated in tyro ${ }^{-1-}$ mice in vivo, we found similar differences in cultured osteoclast in vitro. Expression of RANK and OSCAR was significantly lower in tyro3 $3^{-/-}$cultures than in wild-type controls (figure 4C). Moreover, induction of RANK and OSCAR expression by TNF- $\alpha$ was seen only in wild-type but not in tyro3-deficient cells (figure 4D).

\section{Direct and indirect induction of human osteoclastogenesis by GAS6 in Tyro3-dependent manner}

We next wanted to translate these findings to human disease and tested whether GAS6-Tyro3 interactions have an impact on human osteoclast differentiation. When human osteoclast precursor cells were exposed to GAS6, a dose-dependent stimulation of osteoclast differentiation was observed (figure 5A,B). This effect was particularly pronounced when cells were cultured under suboptimal concentrations of RANKL. GAS6 also induced osteoclastogenesis in monocytes from wild-type but not 
a.

$$
\text { Tyro3+/+ }
$$

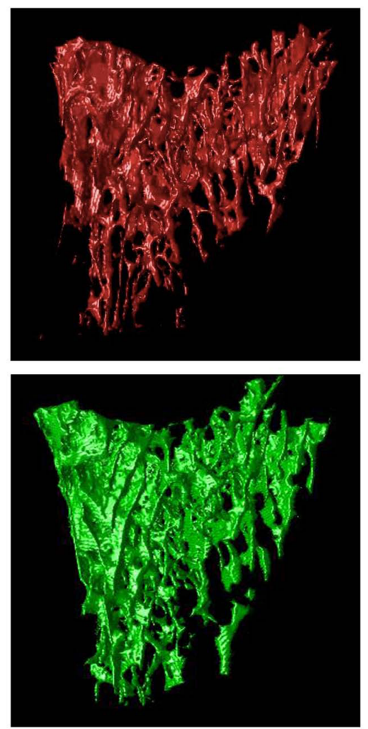

Tyro3-/-

C.
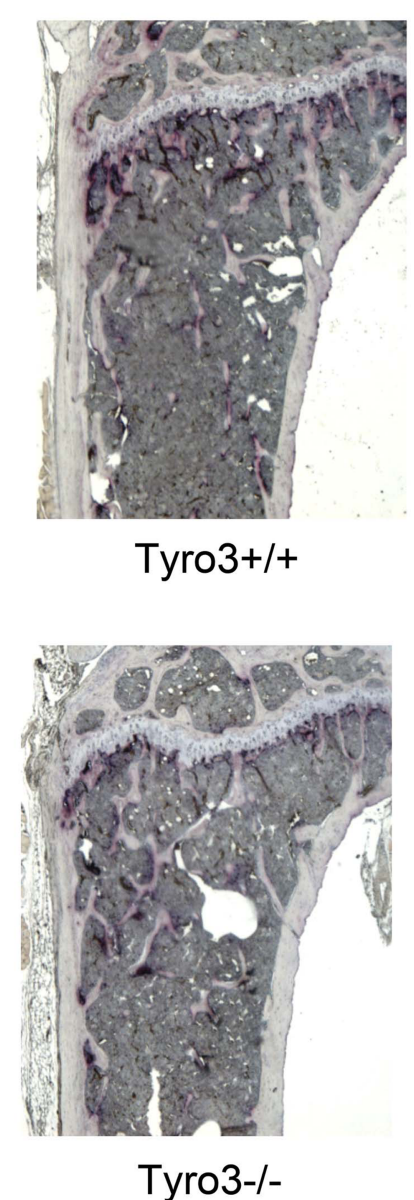

b.

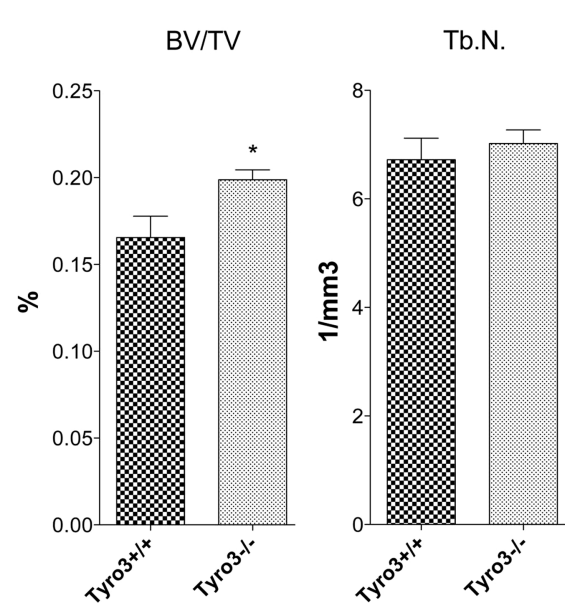

Tb.Th.

Tb.S.

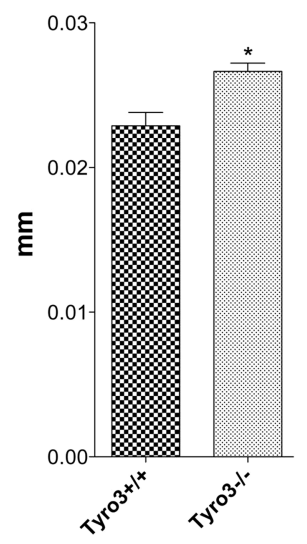

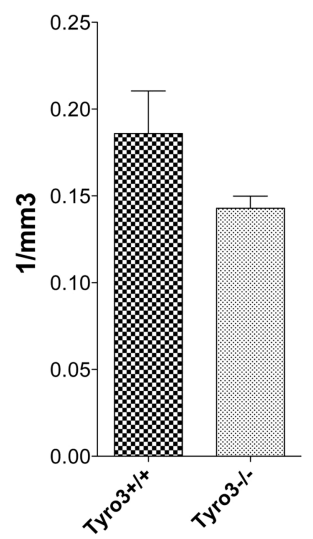

d.
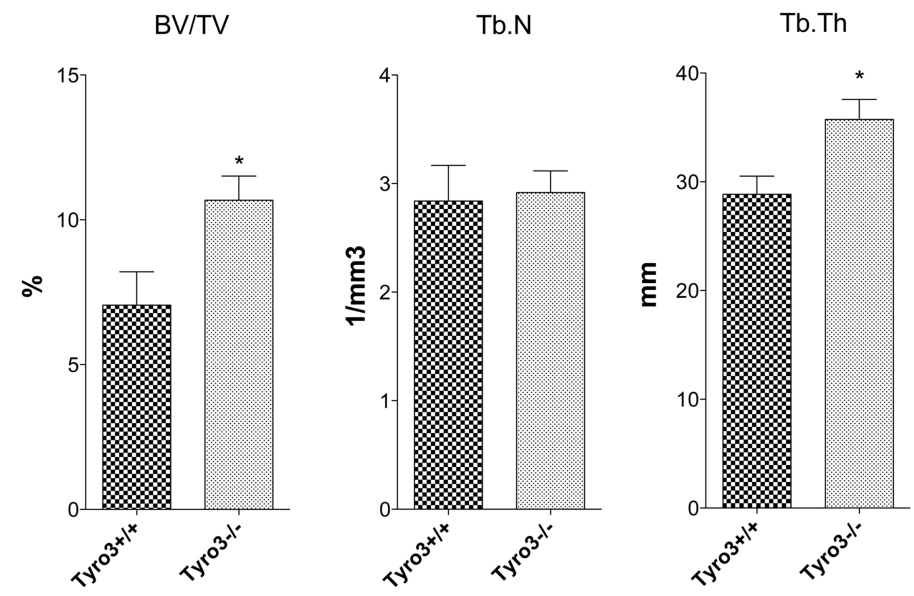

OcN/B.Pm

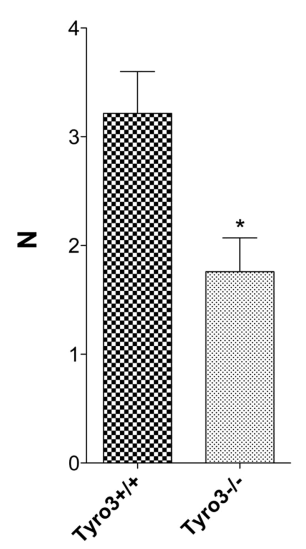

ObN/B.Pm

MAR
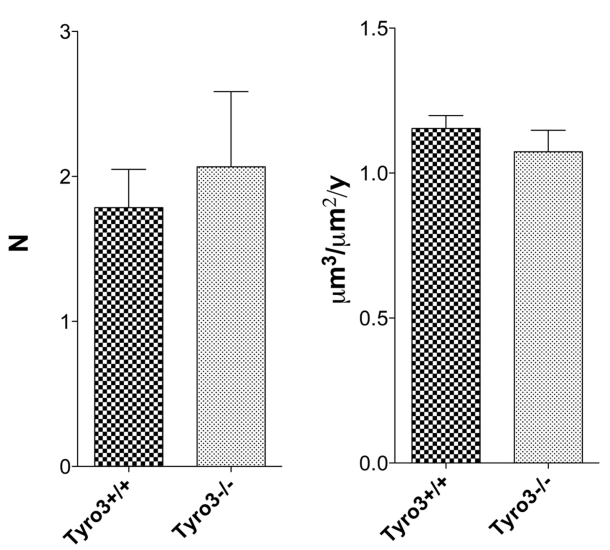

Figure 1 High bone mass due to low osteoclast numbers in tyro3 ${ }^{-1-}$ mice (A) Microphotographs showing trabecular structure of the tibial bone of tyro $^{-I-}$ mice and littermate controls assessed by micro-CT. (B) Results from micro-CT in tyro $3^{-l-}$ mice $(\mathrm{N}=10)$ and littermate controls $(\mathrm{N}=10)$ showing bone volume per total volume (BV/TV), trabecular thickness (Tb.Th) and number (Tb.N) and separation (Tb.S). (C) Microphotographs show trabecular structure of the tibial bone of tyro $3^{-I-}$ mice and littermate controls. Data are the mean \pm SEM. Asterisks indicate significant differences $(p<0.05)$. (D) Results from histomorphometry of tyro3 ${ }^{-1-}$ mice $(N=5)$ and littermate controls $(N=5)$; the following parameters were analysed: BV/TV, trabecular number (Tb.N), trabecular thickness (Tb.Th), number of osteoclast per bone perimeter (N.Oc/B.Pm), number of osteoblast per bone perimeter (N.Ob/B.Pm) and mineral apposition rate (MAR). 


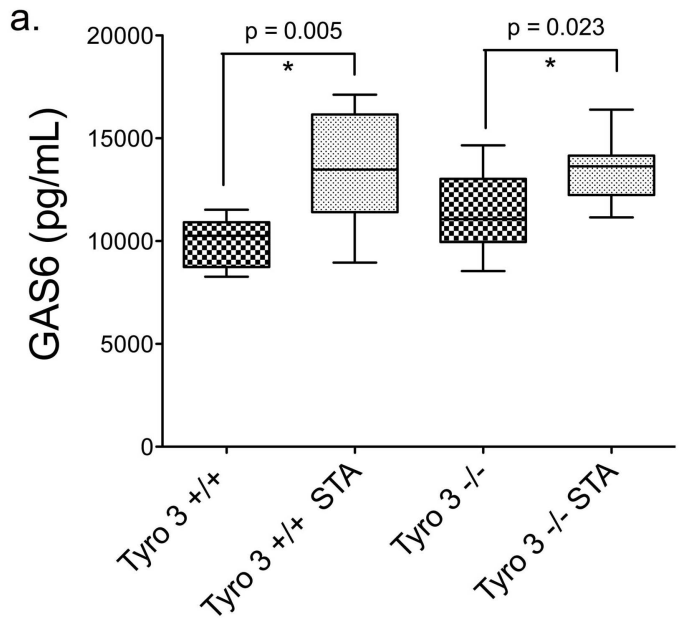

b.

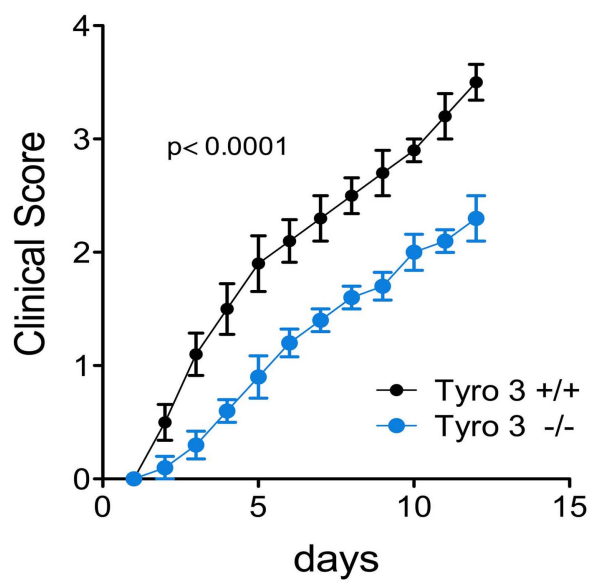

Figure 2 Role of the GAS6-Tyro3 pathway in arthritis (A) Circulating levels of GAS6 in tyro $3^{-1-}$ mice $(\mathrm{N}=10)$ and littermate controls $(\mathrm{N}=10)$ with and without induction of arthritis. Data are the mean \pm SEM. Asterisks indicate significant differences $(p<0.05)$. (B) Clinical evaluation of joint swelling of control mice $(n=10)$ and Tyro3 ${ }^{-1-}$ mice $(n=10)$ induced for arthritis by serum transfer.

in Tyro3-deficient mice (data not shown); however, it was not able to promote osteoclastogenesis in the absence of RANKL either in murine or in human cells. The pro-osteoclastogenic effect of GAS6 in human osteoclasts could be dose dependently abolished by addition of its soluble receptor Tyro3:Fc but not $\mathrm{Axl}$ :Fc (figure 5C). Aside from promoting osteoclastogenesis GAS6 also stimulated the proliferation of synovial fibroblasts. Human synovial fibroblasts did show better proliferation when exposed to GAS6 (figure 5D). This effect could be overcome by adding Tyro3:Fc or Axl:Fc, suggesting both receptors being involved in the autocrine stimulation of fibroblasts. Interestingly, soluble Axl (Axl:Fc) yielded similar effects to Tyro3:Fc on fibroblasts but did not affect GAS6-induced osteoclastogenesis (figure 5E). Finally, we assessed whether induction of osteoclastogenesis by synovial fibroblasts depends on GAS6. We performed coculture experiments with synovial fibroblasts, acting as a source for RANKL ${ }^{16}$ and monocytes. We could show that addition of Tyro3:Fc dose dependently inhibited osteoclastogenesis by synovial fibroblasts, suggesting that Gas6-Tyro3 interactions are essential for fibroblast-mediated stimulation of osteoclast differentiation (figure $5 \mathrm{~F}$ ).

\section{DISCUSSION}

The knowledge on the role of Gas6 and Tyro3 in bone biology was so far confined to in vitro observations. Three independent studies in the late 1990s and early 2000s have shown that the Gas6/Tyro3 system induces the differentiation of osteoclasts. ${ }^{11-13}$ Two of these studies have also shown that activation of the ERK mitogen-activated protein kinase pathway is an essential step for induction of osteoclast differentiation upon binding of Gas6 to Tyro3. ${ }^{12}{ }^{13}$ However, these findings have not prompted investigators to assess the role of Tyro3 in bone homoeostasis in vivo and to search for potential relevance of the Gas6/Tyro3 system in pathological bone loss. In this study, we have shown that deletion of tyro3, which disrupts Gas6-Tyro3 interaction, results in (1) significant increase of bone mass and (2) protection from bone loss during arthritis.

Interestingly, the Tyro3 ligand, GAS6, has been originally described as a gene upregulated in fibroblasts. ${ }^{19}$ GAS6 facilitates the proliferation and survival of fibroblasts, which may partly explain the mechanism of synovial hyperplasia in RA, which is characterised by the accumulation of activated synovial fibroblasts associated with cartilage and bone destruction in RA. ${ }^{14} 18{ }^{23}$ Indeed, we found that the anti-inflammatory effect of Tyro3 deletion is based on the inhibition of synovial hyperplasia. In addition, our experiments showed that GAS6 induces the proliferation of human synovial fibroblasts by interaction with its receptors Tyro3 and Axl. In this context, it is interesting that O'Donnell and colleagues have shown that GAS6 is upregulated in the synovium of patients with RA. ${ }^{9}$ Expression of GAS6 by human synovial fibroblasts could thus represent a link between synovial hyperplasia and bone and cartilage destruction in RA. Our data support this concept by showing that inflammatory bone loss is regulated by Gas6 binding to Tyro3 expressed on the surface of monocyte/osteoclast lineage cells. In addition, our human data clearly showed that GAS6 induce osteoclast differentiation in human monocytes in a Tyro3-dependent manner and also that fibroblasts isolated from the joints of patients with RA support osteoclast differentiation in GAS6-Tyro3-dependent manner.

Earlier studies by Loeser and colleagues ${ }^{20}$ have also suggested a role of Gas6-Tyro3 interaction in chondrocytes. Gas6 can stimulate chondrocyte proliferation and survival, which would be beneficial in arthritis. However, in our studies, we could not observe increased cartilage damage in Tyro3-deficient mice, but rather a protection from cartilage damage in conjunction with arthritis. Abrogation of GAS6-Tyro3 interaction may not essentially hamper chondrocyte survival, but allow protection from articular cartilage damage due to less synovial hyperplasia as well as due to the preservation of bone. Aside from bonecartilage interactions with the synovium, our study opens new possibilities for future research on synovial hyperplasia in arthritis: (1) Stanczyk and colleagues ${ }^{24}$ have shown that synovial fibroblast activation is at least in part controlled by microRNAs (eg, microRNA-203). It remains to be determined whether GAS6 affect the expression of these microRNAs, and thereby, control proliferation and activation of these cells. Interactions of GAS6 with certain microRNAs occurs in cancer. $^{25}$ (2) Mesenchymal stem cells (MSCs) have been described in the synovium $^{26}$ and may represent an important source for proliferating synovial fibroblasts. The effect of GAS6 on MSC is unclear, but it has been shown that GAS6 inhibits the differentiation of these cells into mature chondrocytes. ${ }^{27} 28$ GAS6 could maintain the MSC pool as a source for synovial hyperplasia. (3) Finally, previous studies suggest that GAS6 influences the 
a.
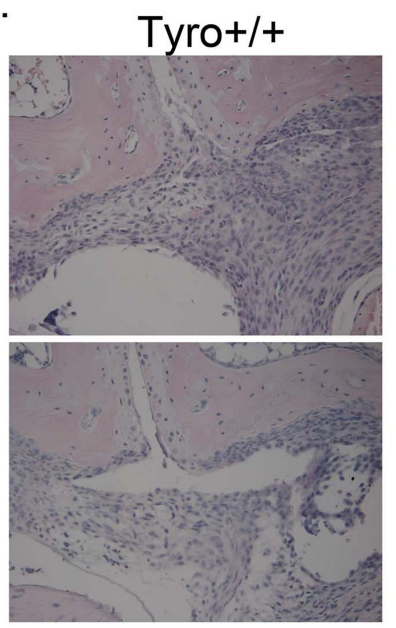

Tyro-/-

d.

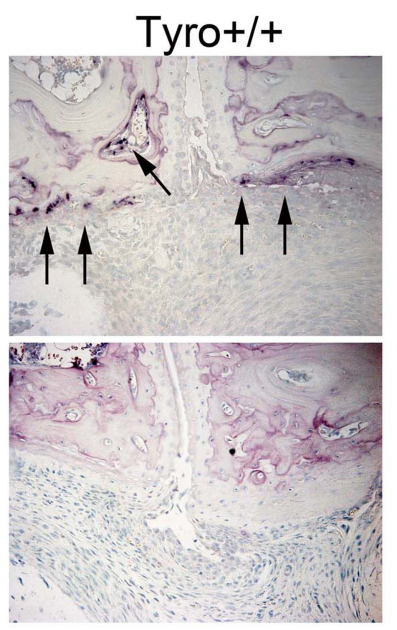

Tyro-/-

g.

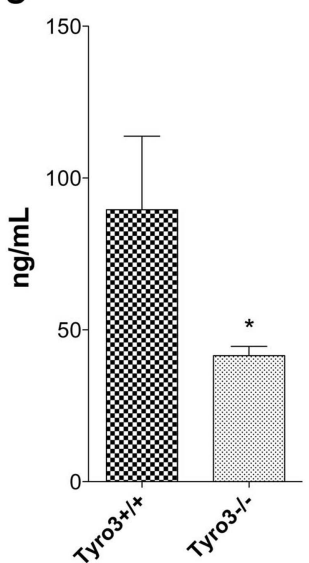

Tyro+/+
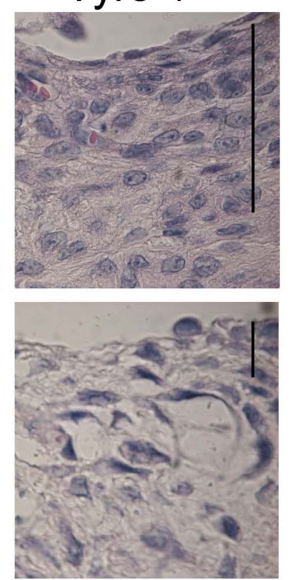

Tyro-/-
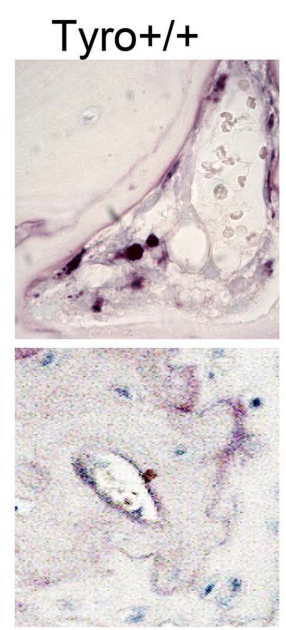

Tyro-/- b.

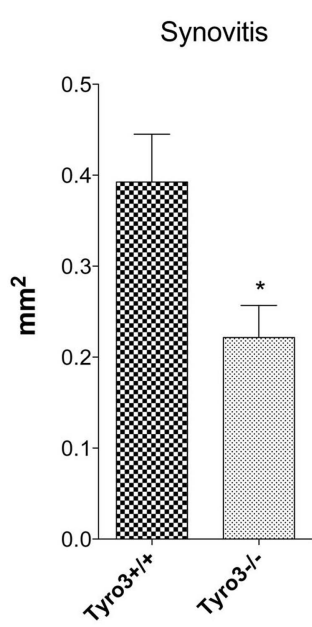

e.

Bone Erosion

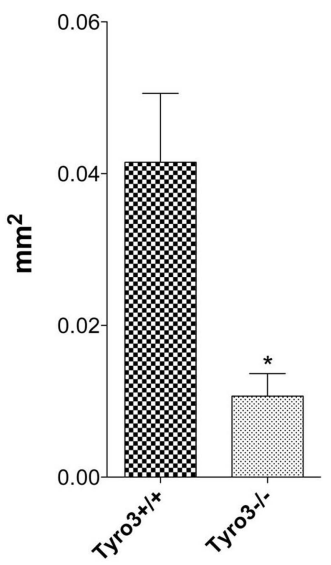

h.

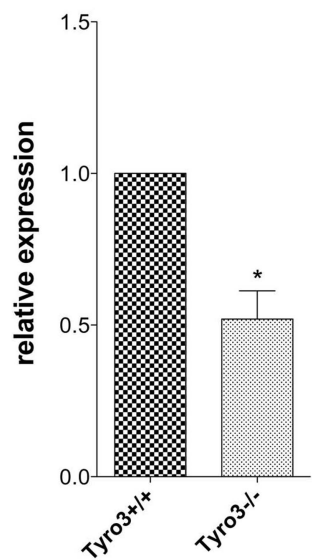

C. Synovial hyperplasia

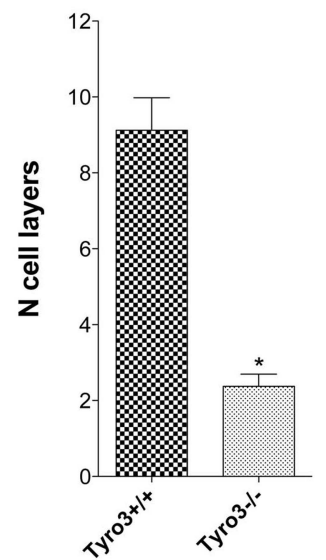

f.

Osteoclasts

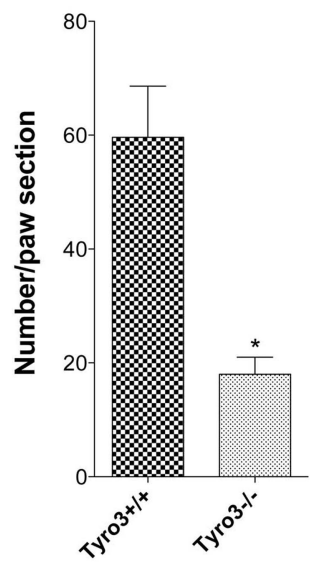

OSCAR

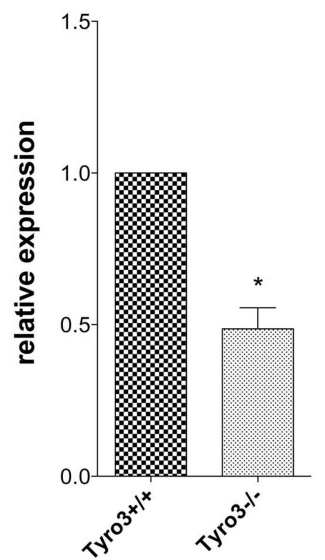

Figure 3 Less synovial hyperplasia and bone erosion in tyro $3^{-/}$mice (A) Microphotographs showing H\&E-stained hind paw section of wild-type littermates and tyro $3^{-1-}$ mice. Left: talonavicular joint (original magnification $\times 100$ ), right: close-up showing the synovial lining (black bars; (original magnification $\times 400$ ); (B) quantification of synovitis and (C) synovial hyperplasia in the two groups (each $n=10$ ); (D) microphotographs showing TRAP-stained hind paw section of wild-type littermates and tyro $3^{-1}$ mice. Left: talonavicular joint (original magnification $\times 100$; black arrows: osteoclasts), right: close-up showing osteoclasts and erosion (original magnification $\times 400)$; (E) quantification of bone erosion and (F) osteoclast numbers in the two groups (each $\mathrm{n=10}$ ); (G) Serum levels of collagen type I cleavage products, a marker for bone resorption in tyro $3^{-l-}$ mice $(\mathrm{N}=10)$ and littermate controls $(\mathrm{N}=10)$ induced for arthritis by serum transfer. $(\mathrm{H})$ Expression of osteoclast receptors OSCAR and RANK in the knee joints of arthritic tyro $3^{-1-}$ mice $(\mathrm{N}=10)$ and littermate controls $(\mathrm{N}=10)$ measured by reverse transcriptase-PCR. Data are the mean $\pm \mathrm{SEM}$. Asterisks indicate significant differences $(\mathrm{p}<0.05)$. 
a.

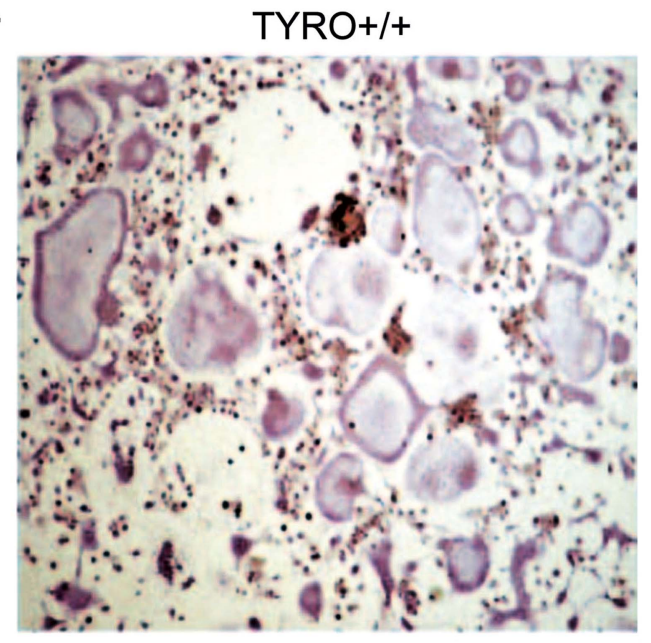

b.

Osteoclast Numbers

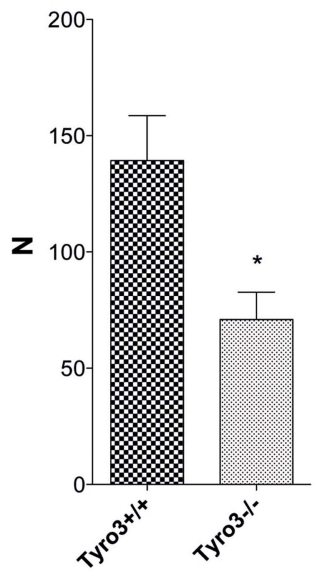

d.

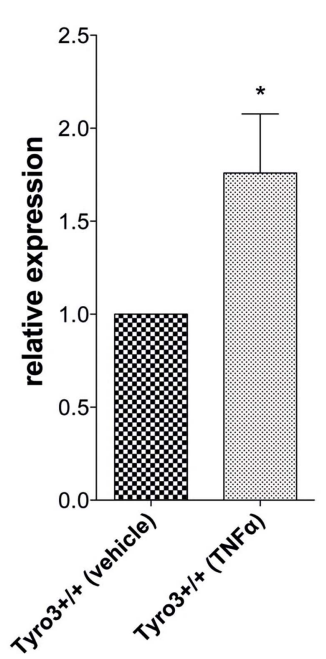

Osteoclast Size

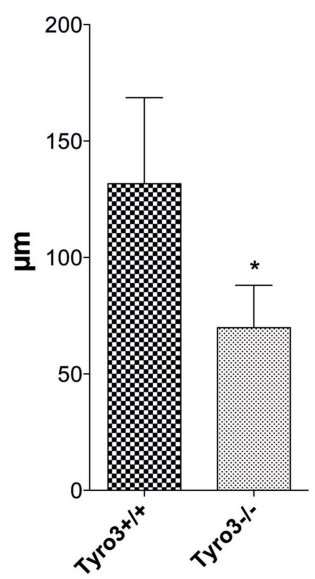

RANK

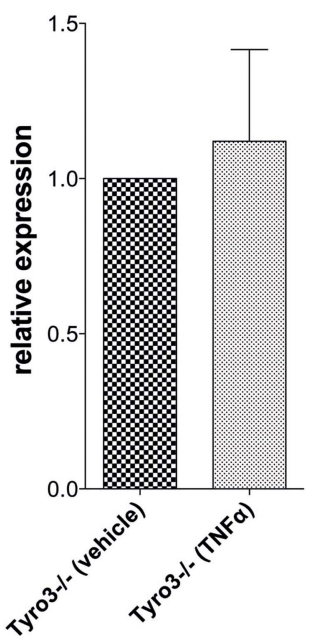

TYRO-I-

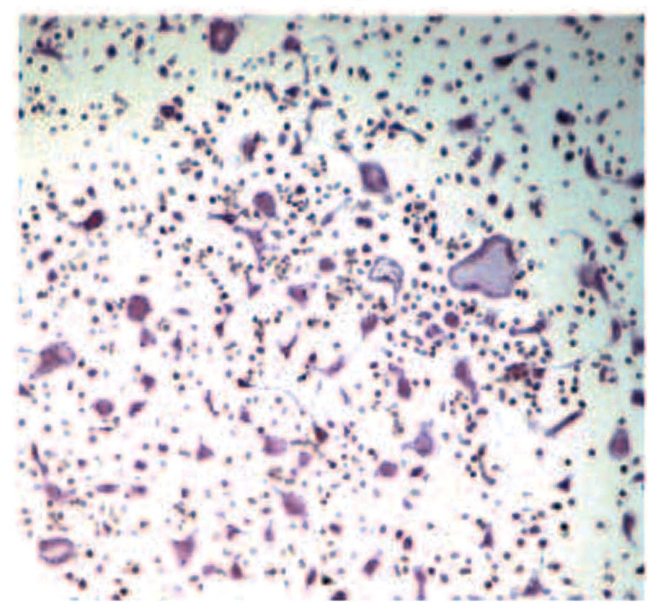

c.

RANK
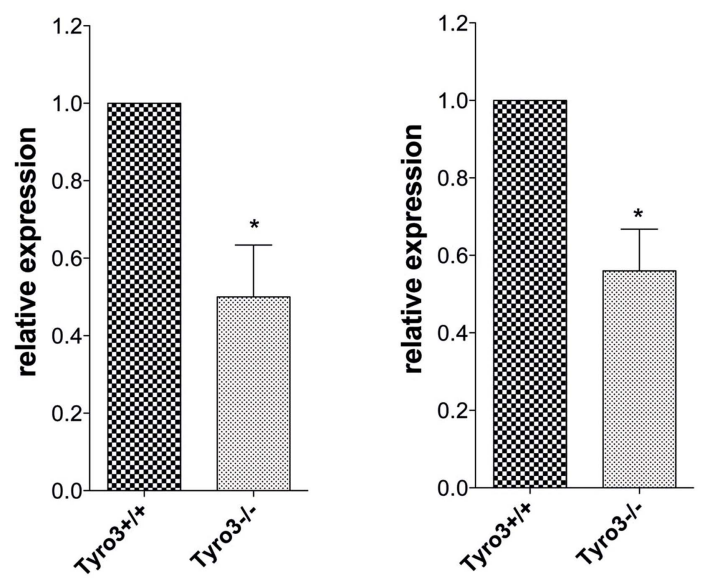

OSCAR
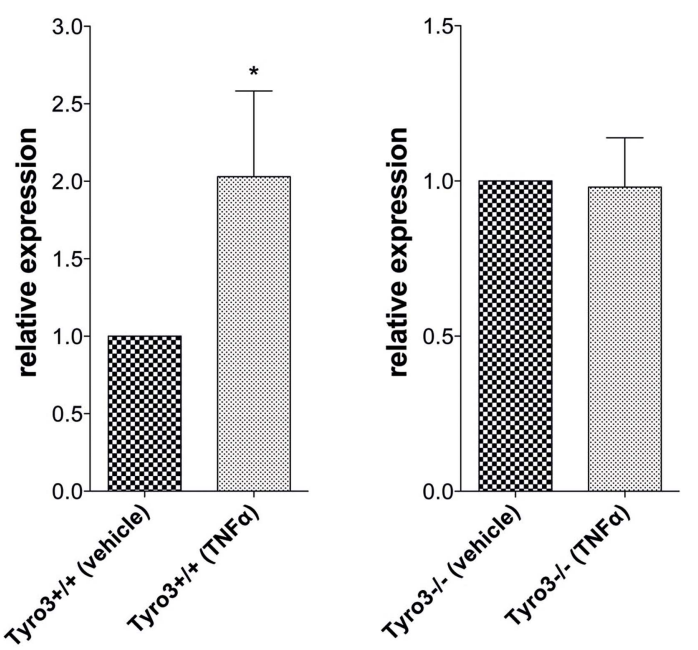

Figure 4 Impaired bone resorption, osteoclast differentiation and gene expression in tyro $3^{-1-}$ mice $(A, B)$ Microphotographs and graphs showing the results of osteoclast differentiation assays from tyro $3^{-1-}$ mice $(\mathrm{N}=10)$ and littermate controls $(\mathrm{N}=10)$. Osteoclasts appear as purple-stained cells TRAP staining. (C) Expression of osteoclast receptors OSCAR and RANK in osteoclast cultures from tyro3 ${ }^{-1-}$ mice $(\mathrm{N}=5)$ and littermate controls $(\mathrm{N}=5)$. (D) Expression of osteoclast receptors OSCAR and RANK in osteoclast cultures from tyro3 $3^{-1-}$ mice $(\mathrm{N}=5)$ and littermate controls $(\mathrm{N}=5)$ with and without exposure to TNF- $\alpha$. Data are the mean \pm SEM. Asterisks indicate significant differences $(p<0.05)$. 
a.

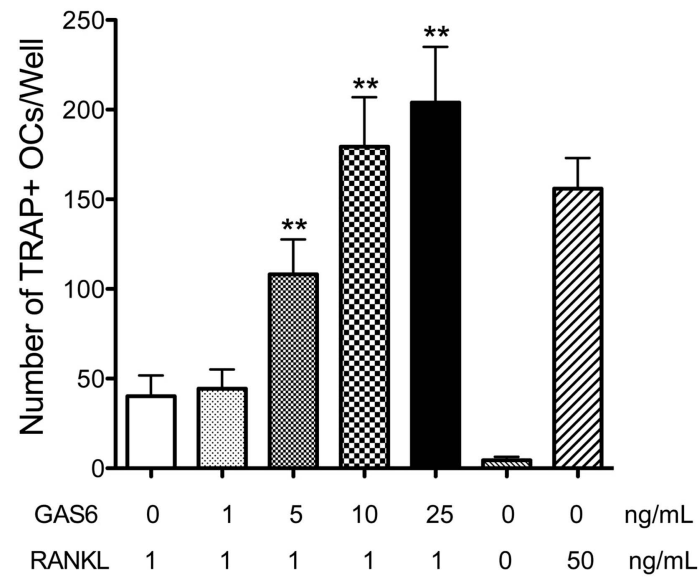

C.

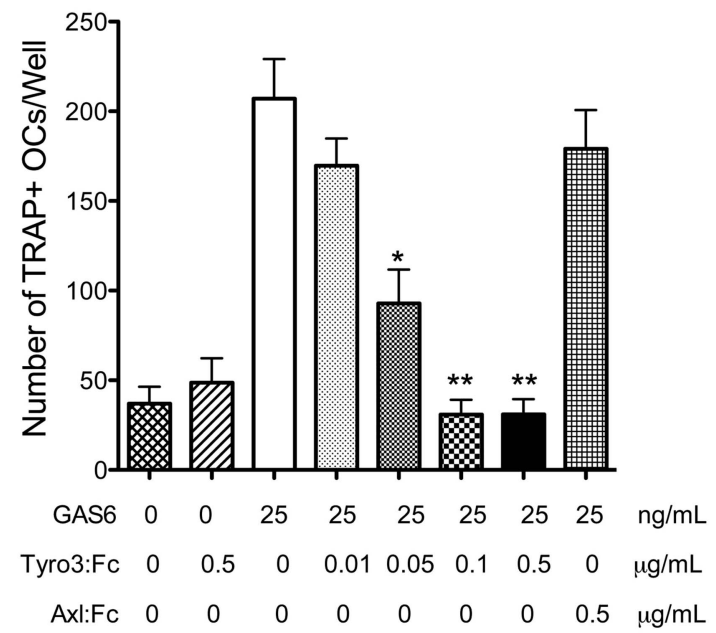

e.

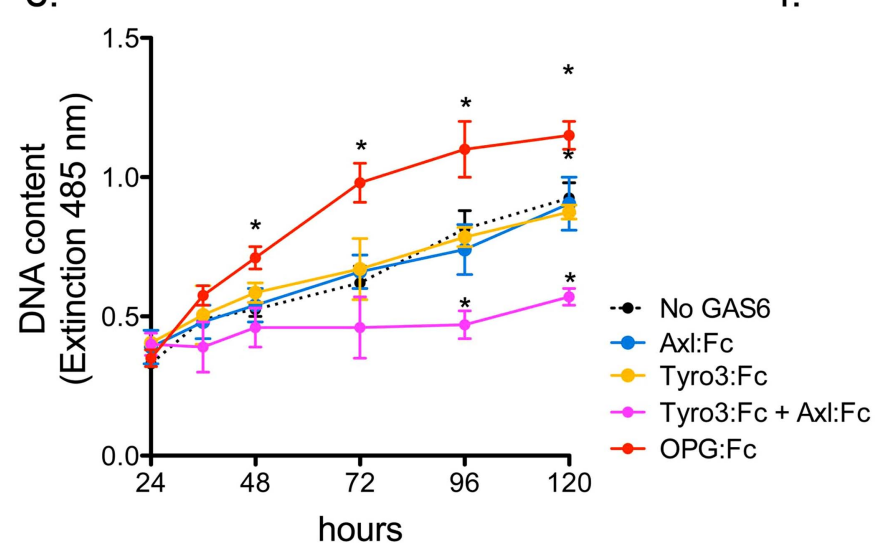

b.

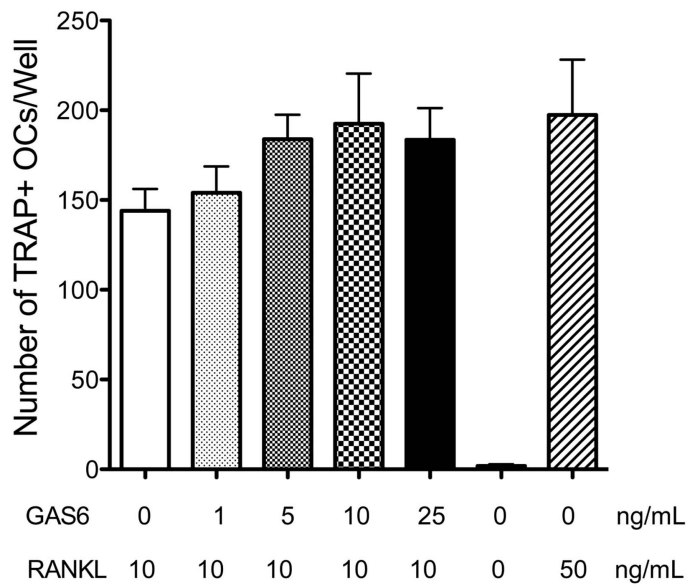

d.

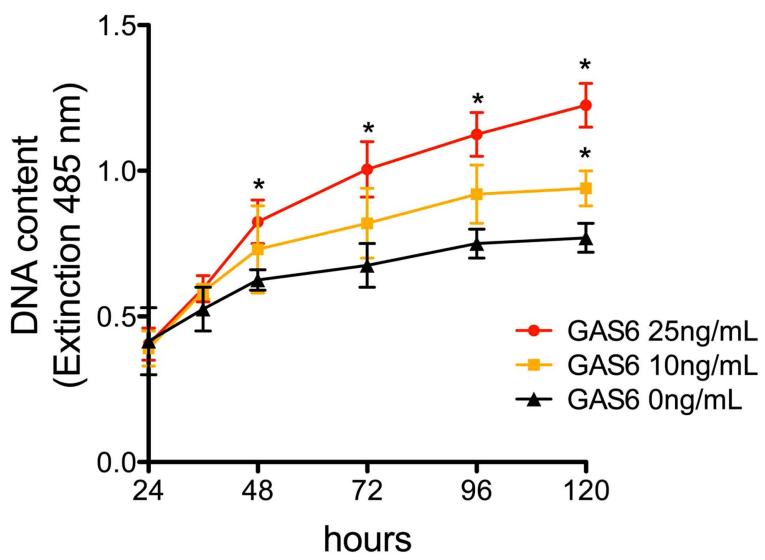

Figure 5 GAS6-induced human osteoclast differentiation and human synovial fibroblast proliferation in Tyro3-dependent manner. (A) Human osteoclast precursors were cultured with $10 \mathrm{ng} / \mathrm{ml} \mathrm{M-CSF}$ and $1 \mathrm{ng} / \mathrm{ml}$ RANKL as well as various amounts of GAS6 for 14 days. (B) Human osteoclast precursors were cultured with $10 \mathrm{ng} / \mathrm{ml} \mathrm{M-CSF}$ and $50 \mathrm{ng} / \mathrm{ml}$ RANKL as well as various amounts of GAS6 for 14 days. (C) Dose-dependent inhibition of GAS6-induced osteoclast differentiation by Tyro3:Fc in the presence of $1 \mathrm{ng} / \mathrm{ml}$ RANKL. (D) Proliferation assay of human synovial fibroblasts exposed to GAS6. (E) Inhibition of GAS6 (25 ng/ml)-induced proliferation of human synovial fibroblasts by Tyro3:Fc $(0.5 \mu \mathrm{g} / \mathrm{ml}$; yellow line) and Axl: Fc $(0.5 \mu \mathrm{g} / \mathrm{ml}$; blue line) and a combination of both agents (each $0.5 \mu \mathrm{g} / \mathrm{ml}$; purple line). Controls were stimulated with $25 \mathrm{ng} / \mathrm{ml}$ GAS6 and Fc-fusion protein not related to the GAS6-Tyro3 pathway (osteoprotegerin (OPG):Fc; $0.5 \mu \mathrm{g} / \mathrm{ml}$; red line) or were left without GAS6 stimulation (black dotted line). (F) Coculture of human synovial fibroblasts and osteoclasts in the presence of various amounts of Tyro3:Fc. OPG:Fc (0.5 $\mu \mathrm{g} / \mathrm{ml})$ was used as positive control for inhibition of osteoclastogenesis. All data are from three independent experiments. Data are the mean $\pm \mathrm{SEM}$. Asterisks indicate significant differences $\left({ }^{*} p<0.05,{ }^{* *} p<0.01\right)$. 


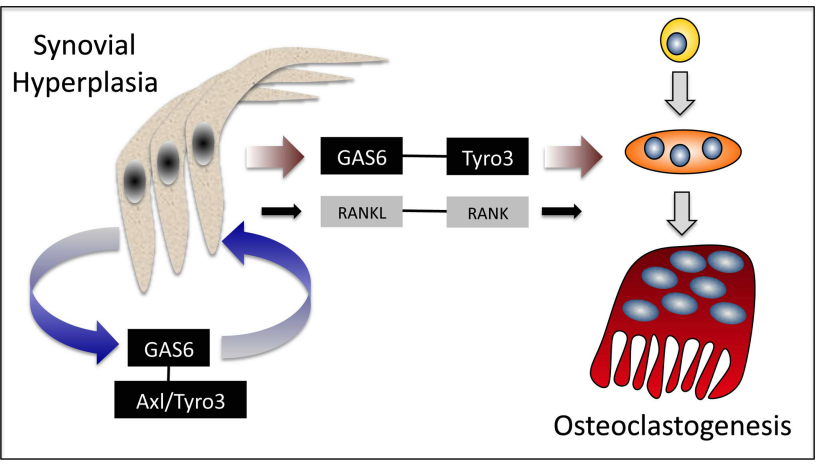

Figure 6 Scheme on the role of GAS6 in synovial hyperplasia and osteoclastogenesis. Synovial fibroblasts proliferate by secreting GAS6 and autocrine stimulation of its receptors Tyro3 and Axl. In addition, GAS6 produced by synovial fibroblasts leads to Tyro3-mediated osteoclast differentiation.

chemoattractive behaviour of fibroblasts, allowing prolonged interactions with haematopoietic cells. ${ }^{29}$ It can be speculated that GAS6 is at least partly responsible for the distinct chemokine and cytokine expression patterns found in synovial fibroblasts. $^{30}$

Strategies that inhibit GAS6 or its receptor Tyro3 could thus emerge as tools to reduce synovial hyperplasia and limit structural damage, particularly in RA. In contrast, diseases with a strong on IL-17/IL-23 signature, such as psoriasis and potentially also spondyloarthritis (SpA), may profit from GAS6 agonists rather than antagonists. van den Brand and colleagues ${ }^{3}$ have shown that GAS6 controls IL-12 and IL-23 production and that fostering GAS6 activity suppresses the expression of these two cytokines and mitigates disease activity in collagen-induced arthritis. Considering that GAS6 acts as a proliferation signal, however, direct neutralisation of IL-17 or IL-23 may be a more feasible strategy to treat disease like psoriasis and SpA, than an indirect approach through GAS6.

In summary, our data suggest that GAS6-Tyro3 ligand-receptor interactions affect bone mass in vivo. GAS6 directly induces osteoclast differentiation, and disruption of GAS6-Tyro3 interaction leads to impaired osteoclast differentiation and higher bone mass. This mechanism appears being particularly relevant in diseases with high local production of GAS6, such as RA, where synovial fibroblast produce this survival-associated and proliferation-associated protein, which links synovial hyperplasia with osteoclastogenesis (figure 6).

Acknowledgements This study was supported by the Deutsche Forschungsgemeinschaft (SPP1468-Immunobone), the Bundesministerium für Bildung und Forschung (BMBF; project Ankyloss), the Marie Curie Training Network grant Osteoimmune, the Masterswitch and Euroteam project of the European Union and the IMI funded project BTCure. We thank Isabel Schmidt, Barbara Happich and Martin Steffen for technical assistance.

Contributors GR-H, YZ, AD, TB, KE and EN collected the data. UM-L, YL, JZ and GS designed the study. GR-H and GS wrote the manuscript.

Competing interests None.

Provenance and peer review Not commissioned; externally peer reviewed.

\section{REFERENCES}

1 Stitt TN, Conn G, Gore $M$, et al. The anticoagulation factor protein $S$ and its relative, Gas 6, are ligands for the Tyro3/Axl family of receptor tyrosine kinases. Cell 1995;80:661-70.
2 Linger RM, Cohen RA, Cummings CT, et al. Mer or Axl receptor tyrosine kinase inhibition promotes apoptosis, blocks growth and enhances chemosensitivity of human non-small cell lung cancer. Oncogene 2012.

3 van den Brand BT, Abdollahi-Roodsaz S, Vermeij EA, et al. Therapeutic efficacy of Tyro3, Axl, and MerTK agonists in collagen-induced arthritis. Arthritis Rheum 2012

4 Lu Q, Lemke G. Homeostatic regulation of the immune system by receptor tyrosine kinases of the Tyro3 family. Science 2001;293:306-11.

5 Sharif MN, Sosic D, Rothlin CV, et al. Twist mediates suppression of inflammation by type I IFNs and Axl. J Exp Med 2006;203:1891-901.

6 Angelillo-Scherrer A, Burnier L, Lambrechts D, et al. Role of Gas6 in erythropoiesis and anemia in mice. J Clin Invest 2008;118:583-96.

7 Angelillo-Scherrer A, Burnier L, Flores N, et al. Role of Gas6 receptors in platelet signaling during thrombus stabilization and implications for antithrombotic therapy. J Clin Invest 2005;115:237-46.

8 Verma A, Warner SL, Vankayalapati $\mathrm{H}$, et al. Targeting Axl and Mer kinases in cancer. Mol Cancer Ther 10:1763-73.

9 Katagiri M, Hakeda Y, Chikazu D, et al. Mechanism of stimulation of osteoclastic bone resorption through Gas6/Tyro3, a receptor tyrosine kinase signaling, in mouse osteoclasts. J Biol Chem 2001;276:7376-82.

10 Kawaguchi H, Katagiri M, Chikazu D. Osteoclastic bone resorption through receptor tyrosine kinase and extracellular signal-regulated kinase signaling in mature osteoclasts. Mod Rheumatol 2004;14:1-5.

11 O'Donnell K, Harkes IC, Dougherty L, et al. Expression of receptor tyrosine kinase Axl and its ligand Gas6 in rheumatoid arthritis: evidence for a novel endothelial cell survival pathway. Am J Pathol 1999;154:1171-80.

12 Ekman C, Linder A, Akesson P, et al. Plasma concentrations of Gas6 (growth arrest specific protein 6) and its soluble tyrosine kinase receptor sAxl in sepsis and systemic inflammatory response syndromes. Crit Care 14:R158.

13 Nakamura YS, Hakeda Y, Takakura N, et al. Tyro3 receptor tyrosine kinase and its ligand, Gas6, stimulate the function of osteoclasts. Stem Cells 1998;16:229-38.

14 Schett G, David JP. The multiple faces of autoimmune-mediated bone loss. Nat Rev Endocrinol 2010;6:698-706.

15 Harre $\mathrm{U}$, Georgess D, Bang H, et al. Induction of osteoclastogenesis and bone loss by human autoantibodies against citrullinated vimentin. J Clin Invest 2012;122:1791-802.

16 Gravallese EM, Harada Y, Wang JT, et al. Identification of cell types responsible for bone resorption in rheumatoid arthritis and juvenile rheumatoid arthritis. Am J Pathol 1998;152:943-51.

17 Gravallese EM, Manning C, Tsay A, et al. Synovial tissue in rheumatoid arthritis is a source of osteoclast differentiation factor. Arthritis Rheum 2000;43:250-8.

18 Mclnnes IB, Schett G. Cytokines in the pathogenesis of rheumatoid arthritis. Nt Rev Immunol 2007;7:429-42.

19 Goruppi S, Ruaro E, Schneider C. Gas6, the ligand of Axl tyrosine kinase receptor, has mitogenic and survival activities for serum starved NIH3T3 fibroblasts. Oncogene 1996;12:471-80.

20 Loeser RF, Varnum BC, Carlson CS, et al. Human chondrocyte expression of growth-arrest-specific gene 6 and the tyrosine kinase receptor axl: potential role in autocrine signaling in cartilage. Arthritis Rheum 1997;40:1455-65.

21 Maccioni M, Zeder-Lutz G, Huang $\mathrm{H}$, et al. Arthritogenic monoclonal antibodies from K/BxN mice. J Exp Med 2002;195:1071-7.

22 Parfitt AM, Drezner MK, Glorieux FH, et al. Bone histomorphometry: standardization of nomenclature, symbols, and units. Report of the ASBMR Histomorphometry Nomenclature Committee. J Bone Miner Res 1987;2:595-610.

23 Mueller-Ladner M, Gay S. MMPs and rheumatoid synovial fibroblasts: Siamese twins in joint destruction? Ann Rheum Dis 2002;61:957-9.

24 Stanczyk J, Ospelt C, Karouzakis E, et al. Altered expression of microRNA-203 in rheumatoid arthritis synovial fibroblasts and its role in fibroblast activation. Arthritis Rheum 2011;63:373-81.

25 Png KJ, Halberg N, Yoshida M, et al. A microRNA regulon that mediates endothelial recruitment and metastasis by cancer cells. Nature 2011;481:190-4.

26 Djouad F, Bony C, Häupl T, et al. Transcriptional profiles discriminate bone marrow-derived and synovium-derived mesenchymal stem cells. Arthritis Res Ther 2005; 7:R1304-15.

27 Filer A, Bik M, Parsonage GN, et al. Galectin 3 induces a distinctive pattern of cytokine and chemokine production in rheumatoid synovial fibroblasts via selective signaling pathways. Arthritis Rheum 2009;60:1604-14.

28 Motomura H, Niimi H, Sugimori K, et al. Gas6, a new regulator of chondrogenic differentiation from mesenchymal cells. Biochem Biophys Res Commun 2007:357:997-1003.

29 Wang J, Qiao Y, Sun F, et al. Identification and characterization of mouse Gas6 promoter. Biochem Biophys Res Commun 2008;371:567-72.

30 Dormady SP, Zhang XM, Basch RS. Hematopoietic progenitor cells grow on 3 T3 fibroblast monolayers that overexpress growth arrest-specific gene-6 (GAS6). Proc Natl Acad Sci USA 2000;97:12260-5. 


\section{A RD Deletion of the receptor tyrosine kinase Tyro3} inhibits synovial hyperplasia and bone damage in arthritis

Gisela Ruiz-Heiland, Yi Zhao, Anja Derer, Tobias Braun, Klaus Engelke, Elena Neumann, Ulf Mueller-Ladner, Yi Liu, Jochen Zwerina and Georg Schett

Ann Rheum Dis 2014 73: 771-779 originally published online April 30, 2013

doi: 10.1136/annrheumdis-2012-202907

Updated information and services can be found at:

http://ard.bmj.com/content/73/4/771

These include:

Supplementary Material

References

Email alerting service
Supplementary material can be found at:

http://ard.bmj.com/content/suppl/2013/04/29/annrheumdis-2012-2029 07.DC1.html

This article cites 26 articles, 6 of which you can access for free at: http://ard.bmj.com/content/73/4/771\#BIBL

Receive free email alerts when new articles cite this article. Sign up in the box at the top right corner of the online article.

Topic
Collections

Articles on similar topics can be found in the following collections

Degenerative joint disease (4622)

Musculoskeletal syndromes (4931)

\section{Notes}

To request permissions go to:

http://group.bmj.com/group/rights-licensing/permissions

To order reprints go to:

http://journals.bmj.com/cgi/reprintform

To subscribe to BMJ go to:

http://group.bmj.com/subscribe/ 